\title{
A Short Survey on MRI Brain Detection
}

\author{
Si-Yuan Lu*, Xing-Xing Zhou, Guang-Shuai Zhang \\ School of Computer Science and Technology \\ Nanjing Normal University \\ Nanjing, Jiangsu, 210023, China
}

\author{
Ling Wei \\ School of Electronic Information\& Electrical \\ Engineering \\ Shanghai Jiaotong University \\ Shanghai 200030, China
}

\begin{abstract}
Automated and accurate classification of MR brain images is of importance for the analysis. Recently researchers have proposed many different and innovative methods to improve upon this technology. This review focused on discussing the strength and limitations of earlier proposed classification techniques. Besides analyzing these literature, the paper also provides a critical evaluation of the surveyed literature which reveals new hotspots of research.
\end{abstract}

Keywords-magnetic resonance imaging; classification;computer-aideddiagnosis

\section{INTRODUCTION}

Magnetic resonance imaging (MRI) is capable of producing highquality images of the anatomical structures of the human body (especially the brain), while it is a low-risk, fast, non-invasive imaging technique.The rich information it provides is of great help for clinical diagnosis and biomedical research [1]. However, because of this huge informationrepository associated with MRIs,employing manual methods to interpret each image is tedious and time consuming, and it is of necessity to develop automateddiagnosis tools to draw quicker and easier inferences from the MRimages. These automated tools can benefit themedical personnel in diagnosis, prognosis, pre-surgical and postsurgical procedures, etc. [2].

Sophisticated signal/image processing techniques and some computational intelligent techniquesare applied to develop diagnostic tools. In one possible methodology, the problem of automaticclassification of normal/pathological subjects, based on brainMRIs, can be configured as a three-stage problem:1) feature extraction; 2) feature reduction; 3) classification [3].

The main contribution of this study is toreview the most recent classification algorithmsand their state-of-the-art. We summarized the advantages anddisadvantages of the reviewed algorithms in tables to provide astructured vision aspects involved in these algorithms.

This paper is organized as follows: Section 2 presents the recent classification algorithms and their experiment results. Section 3 discusses the limitations of these methods, while the conclusion and future work are presented in Section 4.

\section{METHODS AND RESULTS}

Chaplot, Patnaik, and Jagannathan[4] used the approximation coefficients obtained by discrete wavelet transform (DWT), and employed the self-organizing map (SOM) neural network and support vector machine (SVM). The classification percentage of more than $94 \%$ in case of self-organizing maps and $98 \%$ in case of support vector machine.

However, the number of their extracted features reached as high as 4761 which increases the computational time. Meanwhile, their method was only applied to axial T2-weighted imagesat a particular depth inside the brain, and the dataset in their experiment is too small only containing 52 images.

Maitra and Chatterjee [3] employed the Slantlet transform, which is an improved version of DWT for feature extraction. Their feature vector of each image is created by considering the magnitudes of Slantlet transform outputs corresponding to six spatial positions chosen according to a specific logic. Then, they used the common back-propagation neural network (BPNN).

The system they developed could achieve an excellent classification accuracy of $100 \%$, while the number of the features for the classifierinput is only six, which obviouslylightened computational burden.However, their method was only tested on AD.

El-Dahshan, Hosny, and Salem [5] extracted the approximation and detail coefficients of 3-level DWT, reduced the coefficients by principal component analysis (PCA), and used feed-forward back-propagation artificial neural network (FP-ANN) and K-nearest neighbor (KNN) classifiers.

The FP-ANN and KNN achieved accuracy of 97\% and $98 \%$, respectively. However, whenever the image database increased, a fresh training is required for the proposed system before it works again,which is inconvenient in practical use, and the classification performance can be improved.

Zhang, $\mathrm{Wu}$, and Wang[6] proposed using DWT for feature extraction, PCA for feature reduction, and FNN with scaled chaotic artificial bee colony (SCABC) as classifier. Based on it, Zhang, Dong, Wu, and Wang [7] suggested to replace SCABC with scaled conjugate gradient (SCG) method. Both algorithms achieved 100\% accuracy, and the computation time per image is only 0.0451 s.However, the dataset is too small only containing 66 images, and the requirement for 
computationtime could be decreasedif they used advanced wavelet transformssuch as lift-up wavelet.

Zhang and $\mathrm{Wu}[8]$ proposed to use kernel SVM as classification method, and found the GRB kernel achieved the highest accuracy of $99.38 \%$. The prediction time of a $256 \times 256$ image was only 0.0448 s.

Future scope of their paper is that, they'll test novel kernels to increase the classification accuracy. Meanwhile, multi-classificationcan also be explored, which focuses onspecific disorders utilizing brain MRI.

Saritha, Joseph, and Mathew[9] proposed a novel feature of wavelet-entropy (WE), employed spider-web-plot (SWP) to further reduce features, and used the probabilistic neural network (PNN). The classification accuracy was found to be $100 \%$ on a 75-image dataset with four diseases.

The limitation of their work is that oncethere is an increase in image database, fresh training is required. The size of dataset was small and the number of categories of diseases is too little. The efficiency of the developed system could be improved, sinceZhang, Dong, Ji, and Wang [10] suggested that removing spider-web-plot yielded the same classification performance later.

Das, Chowdhury, and Kundu[2] proposed to use a hybrid method for classification. First they employed Ripplet transform (RT) to extract features from MRIs.And then the feature of Magnetic Resonance Image has been reducedby using Principal Component Analysis (PCA). Afterwards, they developed a classifier based on least square SVM (LS-SVM). Five-fold cross-validation was utilized to avoidoverfitting.

With this combination, theynot only achieved higher feature reduction, but also acquired superiorperformance, which showed high classification accuracy of $99.39 \%$ over a 255-image dataset. However, the time requirement for feature extraction through RT is slightly greater than DWT.

Zhang, Wang, Ji, and Dong[11] suggested a hybrid system, integrating DWT, PCA, PSO,KSVM, and CV, to identify normal MR brains fromabnormal MR brains. The proposed method first employed digital wavelet transform to extract features, and then used principal componentanalysis (PCA) to reduce the feature space. Afterwards, they constructed a kernel support vector machine (KSVM) with RBF kernel,using particle swarm optimization (PSO) to optimize the parameters $\mathrm{C}$ and $\sigma$. Fivefold cross-validation was utilized to avoidoverfitting.

The classification result on a 90-image database achieved $97.11 \%$ accuracy, which shows that the PSO is more effective to findthe optimal values than random selection method. Theclassification capability of KSVMcan be improved by integrating PSO. However, advanced wavelet transforms, such as the lift-up wavelet, can be helpful to accelerate the computation time.

Kalbkhani, Shayesteh, and Zali-Vargahan[12] modelled the detail coefficients of 2-level DWT by generalized autoregressive conditional heteroscedasticity (GARCH) statistical model, and the parameters of GARCH model are considered as the primary feature vector. Their classifier was chosen as KNN and SVM models.

KNNand SVM classifiers achieved 97.62\% and 98.21\% accuracy for theeight-class classification, respectively.
Also, for two-class classification both classifiers achieve $100 \%$ accuracy. Considering that the problem is multi-classification, and the number of features is relatively low, the result is outstanding. However, the feature extraction process of the system requires relatively more time.

Padma and Sukanesh[13] used combined wavelet statistical texture features, to segment and classify AD benign and malignant tumor slices. First, Two dimensional discretewavelet decomposition is performed on the tumor image to remove the noise. Than a total of 17 features are extracted and six features are selected usingStudent's t test. Finally, the reduced optimal features are used tomodel and train the probabilistic neural network classifier and the classification accuracy is evaluated using $\mathrm{k}$ fold cross validation method.

Their classification model achieved $97.39 \%$ classification accuracy based on SVM tumor segmentation and 2D-DWT preprocessing. However, the limitation ofthis method is that it needs new training for the PNN classifier whenever there is change in the slice data set and itcan't be applied to brain imaging techniques but CT.

El-Dahshan, Mohsen, Revett, and Salem [14] used the feedback pulse-coupled neural network for image segmentation, the DWT for features extraction, the PCA for reducing the dimensionality of the wavelet coefficients, and the FBPNN to classify inputs into normal or abnormal.

The accuracy achieved $99 \%$ on a 101-image dataset. However, if they extract moreefficient features and increase the training dataset, the classification can be more accurate.

Zhang, Wang, and Dong[15] presented a diagnosis method to distinguish Alzheimer's disease (AD) and mild cognitive impairment (MCI) from normal controls, based on structural MR images by kernel SVM Decision Tree. A 5-fold cross validation showed their method yielded $80 \%$ accuracy for these three classes, and the computationtime to predict a new patient is only $0.022 \mathrm{~s}$.

The limitation of their method is thatthe meanings of weights/biases of the kSVM-DT mean can't be understood by ordinary technicians, since the classifier establishes machine-oriented rules instead of human-oriented rules. Another limitation is that the classification accuracy needs to be improved. However, structural MRI may not cover the sufficient information containing the cause of $\mathrm{AD}$.

Zhou, et al. [16] used wavelet-entropy as the feature space, then they employed a Naive Bayes classifier (NBC) classification method. Their results over 64 images showed that the sensitivity of the classifier is $94.50 \%$, the specificity $91.70 \%$, the overall accuracy $92.60 \%$.

However, ordinary technicians have difficulty interpreting the entropy values or theweights/biases of NBC.Meanwhile,multi-disease classification was the future research direction, since their classifier can only perform two-class classification. Finally, the small dataset may introduce error to the reported classificationaccuracy.

Zhang, Dong, Wang, Ji, and Yang[17] used discrete wavelet packet transform (DWPT), and harnessed Tsallis entropy to obtain features from DWPT coefficients. Then, 
they used a generalized eigenvalue proximal SVM (GEPSVM) with RBF kernel.

This combination yielded superior performance, achieving $99.61 \%$ accuracy. However, the limitation was that the classifier was machine-oriented instead of human-oriented. Although machine-orientedclassifiers are better in classification performance than human-oriented classifiers, it is difficult for technicians tounderstand or interpret the meanings of weights/biases of the classifier, which is inconvenient in practical use.And the computation of DWPT is relatively time-consuming.

Yang, et al.[18] selected wavelet-energy as the features, and introduced biogeography-based optimization (BBO) to train the SVM. Their method reached 97.78\% accuracy on 90 T2-weighted MR brain images.
Future scope of their paper is that, they'll replace wavelet-energywith more efficient feature descriptors, suchas scale-invariant features and employ advanced pattern recognition techniques, such as deep learning and RBFNN. Multiple slices can be employed in order to improve theclassification performance. They'll also test whether higher level can lead to better classificationperformance by increasing the decompositionlevel of 2D-DWT.

\section{DISCUSSION}

In this review, we gave an overview of the state of the art in the MRI-based medical image classification methods. It can be easily observed that the method is becoming mature. The characteristics of different methods are summarized in Table1.

\begin{tabular}{|c|c|c|c|c|c|}
\hline No & Author & Year & Method used & Limitation & Accuracy \\
\hline 1 & $\begin{array}{l}\text { Chaplot, Patnaik, } \\
\text { and Jagannathan }\end{array}$ & 2006 & 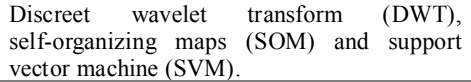 & $\begin{array}{l}\text { The number of the features is too high, and } \\
\text { their method was only applied to axial } \\
\text { T2-weighted images. }\end{array}$ & $\begin{array}{l}94 \% \text { for SOM and } 98 \% \\
\text { for SVM }\end{array}$ \\
\hline 2 & $\begin{array}{l}\text { Maitra and } \\
\text { Chatterjee }\end{array}$ & 2006 & $\begin{array}{l}\text { Slantlet transform (ST) and back propagation } \\
\text { neural network (BPNN). }\end{array}$ & Their method was only tested on $\mathrm{AD}$. & $100 \%$ \\
\hline 3 & $\begin{array}{l}\text { El-Dahshan, } \\
\text { Hosny, and Salem }\end{array}$ & 2010 & $\begin{array}{l}\text { 3-level DWT, principle component analysis } \\
\text { (PCA), feed-forward back-propagation } \\
\text { artificial neural network (FP-ANN) and } \\
\text { K-nearest neighbor (k-NN) }\end{array}$ & $\begin{array}{l}\text { It requires fresh training each time } \\
\text { whenever there is an increase in image } \\
\text { database. }\end{array}$ & $\begin{array}{l}97 \% \text { for } \mathrm{FP}-\mathrm{ANN} \text { and } \\
98 \% \text { for } \mathrm{k}-\mathrm{NN}\end{array}$ \\
\hline 4 & $\begin{array}{l}\text { Zhang, Wu, and } \\
\text { Wang }\end{array}$ & 2011 & $\begin{array}{l}\text { Discreet wavelet transform (DWT), principle } \\
\text { component analysis (PCA) and FNN with } \\
\text { scaled chaotic artificial bee colony (SCABC). }\end{array}$ & $\begin{array}{l}\text { Their method was only applied to } \\
\text { T2-weighted images. }\end{array}$ & $100 \%$ \\
\hline 5 & $\begin{array}{l}\text { Zhang, Wu, and } \\
\text { Wang }\end{array}$ & 2011 & $\begin{array}{l}\text { Discreet wavelet transform (DWT), principle } \\
\text { component analysis (PCA) and back } \\
\text { propagation neural network (BPNN) with } \\
\text { conjugate gradient (SCG). }\end{array}$ & The dataset only contains 66 images. & $100 \%$ \\
\hline 6 & Zhang and $\mathrm{Wu}$ & 2012 & $\begin{array}{l}\text { Discreet wavelet transform (DWT), principle } \\
\text { component analysis (PCA) and kernel support } \\
\text { vector machine (KSVM) with GRB kernel. }\end{array}$ & $\begin{array}{l}\text { The computation time could be accelerated } \\
\text { by using advanced wavelet transforms such } \\
\text { as the lift-up wavelet. }\end{array}$ & $99.38 \%$ \\
\hline 7 & $\begin{array}{l}\text { Saritha, Joseph, } \\
\text { and Mathew }\end{array}$ & 2013 & $\begin{array}{l}\text { Wavelet entropy based spider web plots and } \\
\text { probabilistic neural network. }\end{array}$ & $\begin{array}{l}\text { The size of dataset was small and the } \\
\text { number of categories of diseases is too } \\
\text { little. The same classification performance } \\
\text { can be yielded if the spider-web-plot is } \\
\text { removed. }\end{array}$ & $100 \%$ \\
\hline 8 & $\begin{array}{l}\text { Das, Chowdhury, } \\
\text { and Kundu }\end{array}$ & 2013 & $\begin{array}{l}\text { Ripplet transform (RT), PCA, least square } \\
\text { SVM (LS-SVM), and the } 5 \times 5 \text { CV }\end{array}$ & $\begin{array}{l}\text { The time requirement for feature extraction } \\
\text { through RT is slightly greater than DWT. }\end{array}$ & $99.39 \%$ \\
\hline 9 & $\begin{array}{l}\text { Zhang, Wang, Ji, } \\
\text { and Dong }\end{array}$ & 2013 & $\begin{array}{l}\text { Discreet wavelet transform (DWT), PCA, } \\
\text { particle swarm optimization (PSO), and } \\
\text { KSVM with RBF kernel. }\end{array}$ & $\begin{array}{l}\text { The computation time could be accelerated } \\
\text { by using advanced wavelet transforms such } \\
\text { as the lift-up wavelet. }\end{array}$ & $97.11 \%$ \\
\hline 10 & $\begin{array}{l}\text { Kalbkhani, } \\
\text { Shayesteh, and } \\
\text { Zali-Vargahan }\end{array}$ & 2013 & $\begin{array}{l}\text { Discreet wavelet transform } \text { (DWT), } \\
\text { generalized autoregressive conditional } \\
\text { heteroscedasticity (GARCH), K nearest } \\
\text { neighbor }(\mathrm{KNN}) \text { and support vector machine } \\
(\mathrm{SVM})\end{array}$ & $\begin{array}{l}\text { The feature extraction process requires } \\
\text { relatively more time. }\end{array}$ & $\begin{array}{l}97.62 \% \text { for } \mathrm{k}-\mathrm{NN} \text { and } \\
98.21 \% \text { for } \mathrm{SVM}\end{array}$ \\
\hline 11 & $\begin{array}{l}\text { Padma and } \\
\text { Sukanesh }\end{array}$ & 2014 & $\begin{array}{l}\text { Combined wavelet statistical texture features, } \\
\text { discreet wavelet transform (DWT), support } \\
\text { vector machine (SVM), and Probabilistic } \\
\text { Neural Network (PNN) }\end{array}$ & $\begin{array}{l}\text { It needs new training for the PNN classifier } \\
\text { whenever there is change in the slice data } \\
\text { set and the method can be applied to brain } \\
\text { CT images only. }\end{array}$ & $97.39 \%$ \\
\hline 12 & $\begin{array}{l}\text { El-Dahshan, } \\
\text { Mohsen, Revett, } \\
\text { and Salem }\end{array}$ & 2014 & $\begin{array}{l}\text { Discreet wavelet transform (DWT), PCA, and } \\
\text { feedback pulse-coupled neural network } \\
\text { (FBPNN). }\end{array}$ & $\begin{array}{l}\text { The classification accuracy can be } \\
\text { improved by extracting more efficient } \\
\text { features and increasing the training dataset. }\end{array}$ & $99 \%$ \\
\hline 13 & $\begin{array}{l}\text { Zhang, Wang, and } \\
\text { Dong }\end{array}$ & 2014 & PCA, and kernel SVM Decision Tree. & $\begin{array}{l}\text { The classifier establishes machine-oriented } \\
\text { rules not human-oriented rules. }\end{array}$ & $80 \%$ \\
\hline 14 & Zhou, et al. & 2015 & $\begin{array}{l}\text { 2D-discrete } \\
\text { wavelet-entropy, and Naive Bayes classifier. }\end{array}$ & $\begin{array}{l}\text { It is difficult to interpret the entropy values } \\
\text { or the weights/biases of NBC. }\end{array}$ & $92.60 \%$ \\
\hline 15 & $\begin{array}{l}\text { Zhang, Dong, } \\
\text { Wang, Ji, and } \\
\text { Yang }\end{array}$ & 2015 & $\begin{array}{l}\text { Discrete wavelet packet transform, harnessed } \\
\text { Tsallis entropy, and generalized eigenvalue } \\
\text { proximal SVM (GEPSVM) with RBF } \\
\text { kernel. }\end{array}$ & $\begin{array}{l}\text { The classifier was machine-oriented not } \\
\text { human-oriented. And DWPT takes the most } \\
\text { time during either the offline learning stage } \\
\text { or online prediction stage. }\end{array}$ & $\begin{array}{l}100 \%, \quad 100 \%, \quad \text { and } \\
99.53 \% \text { on Dataset-66, } \\
\text { Dataset-160, and } \\
\text { Dataset-255, } \\
\text { respectively }\end{array}$ \\
\hline 16 & Yang, et al. & 2015 & $\begin{array}{l}\text { Wavelet-Energy, } \\
\text { biogeography-based optimization }(\mathrm{BBO}) \text {. }\end{array}$ & $\begin{array}{l}\text { Wavelet-energy is less efficient than some } \\
\text { other feature descriptors, such as } \\
\text { scale-invariant features. }\end{array}$ & $97.78 \%$ \\
\hline
\end{tabular}




\section{CONCLUSION}

In this paper we have accomplished a short survey ofrecentdetection techniques for MRI brain image.Afterthe analysis of well-known techniques it is clearly shown thevarious methods which can classify MR brain images efficiently andprovide accurate result.As the classification of MR brain images still remains an open problem, there are more novel classification techniques which perform more efficiently and more accurately, such as HPA method [19], 3D-DWT method [20], and eigenbrain [21], which will be included in our future research.

From thesurvey we find thatmost of the latest existingmethods are to develop two-class classifier, while the development of multi-class classifier is still at an initial stage. We believe this will be a hot spot in a foreseeable future.

\section{ACKNOWLEDGMENT}

This paper was supported by NSFC (610011024, 61273243, 51407095), Program of Natural Science Research of Jiangsu Higher Education Institutions (13KJB460011,14KJB520021), Jiangsu Key Laboratory of 3D Printing Equipment and Manufacturing (BM2013006), Key Supporting Science and Technology Program (Industry) of Jiangsu Province (BE2012201, BE2014009-3,BE2013012-2), Special Funds for Scientific and Technological Achievement Transformation Project in Jiangsu Province (BA2013058), and Nanjing Normal University Research Foundation for Talented 2014119XGQ0080).

Scholars

(2013119XGQ0061,

\section{REFERENCES}

[1] Goh, S.; Dong, Z.; Zhang, Y.; DiMauro, S.; Peterson, B.S. Mitochondrial dysfunction as aneurobiological subtype of autism spectrum disorder: Evidence from brain imaging. JAMA Psychiatry2014, 71, 665-671.

[2] Das S, Chowdhury M, Kundu M K. Brain MR image classification using multiscale geometric analysis of ripplet[J]. Progress In Electromagnetics Research, 2013, 137: 1-17.

[3] Maitra M, Chatterjee A. A Slantlet transform based intelligent system for magnetic resonance brain image classification[J]. Biomedical Signal Processing and Control, 2006, 1(4): 299-306.

[4] Chaplot S, Patnaik L M, Jagannathan N R. Classification of magnetic resonance brain images using wavelets as input to support vector machine and neural network[J]. Biomedical Signal Processing and Control, 2006, 1(1): 86-92.

[5] El-Dahshan E S A, Hosny T, Salem A B M. Hybrid intelligent techniques for MRI brain images classification[J]. Digital Signal
Processing, 2010, 20(2): 433-441.

[6] Zhang Y, Wu L, Wang S. Magnetic resonance brain image classification by an improved artificial bee colony algorithm[J]. Progress in Electromagnetics Research, 2011, 116: 65-79.

[7] Zhang Y, Dong Z, Wu L, et al.A hybrid method for MRI brain image classification[J]. Expert Systems with Applications, 2011, 38(8): 10049-10053.

[8] Zhang Y, Wu L. An MR brain images classifier via principal component analysis and kernel support vector machine $[\mathrm{J}]$. Progress in Electromagnetics Research, 2012, 130: 369-388.

[9] Saritha M, Joseph K P, Mathew A T. Classification of MRI brain images using combined wavelet entropy based spider web plots and probabilistic neural network[J]. Pattern Recognition Letters, 2013, 34(16): 2151-2156.

[10] Zhang Y, Dong Z, Ji G, et al. Effect of spider-web-plot in MR brain image classification[J]. Pattern Recognition Letters, 2015, 62: $14-16$.

[11] Zhang Y, Wang S, Ji G, et al. An MR brain images classifier system via particle swarm optimization and kernel support vector machine[J]. The Scientific World Journal, 2013, 2013.

[12] Kalbkhani H, Shayesteh M G, Zali-Vargahan B. Robust algorithm for brain magnetic resonance image (MRI) classification based on GARCH variances series[J]. Biomedical Signal Processing and Control, 2013, 8(6): 909-919.

[13] Padma A, Sukanesh R. Segmentation and classification of brain $\mathrm{CT}$ images using combined wavelet statistical texture features $[\mathrm{J}]$. Arabian Journal for Science and Engineering, 2014, 39(2): 767-776.

[14] El-Dahshan E S A, Mohsen H M, Revett K, et al. Computer-aided diagnosis of human brain tumor through MRI: A survey and a new algorithm[J]. Expert Systems with Applications, 2014, 41(11): 5526-5545.

[15] Zhang Y, Wang S, Dong Z. Classification of Alzheimer disease based on structural magnetic resonance imaging by kernel support vector machine decision tree[J]. Progress In Electromagnetics Research, 2014, 144: 171-184.

[16] Zhou X, Wang S, Xu W, et al. Detection of Pathological Brain in MRI Scanning Based on Wavelet-Entropy and Naive Bayes Classifier[M]//Bioinformatics and Biomedical Engineering. Springer International Publishing, 2015: 201-209.

[17] Zhang Y, Dong Z, Wang S, et al. Preclinical Diagnosis of Magnetic Resonance (MR) Brain Images via Discrete Wavelet Packet Transform with Tsallis Entropy and Generalized Eigenvalue Proximal Support Vector Machine (GEPSVM)[J]. Entropy, 2015, 17(4): 1795-1813.

[18] Yang G, Zhang Y, Yang J, et al. Automated classification of brain images using wavelet-energy and biogeography-based optimization[J]. Multimedia Tools and Applications, 2015: 1-17.

[19] Wang, S., et al., Feed-forward neural network optimized by hybridization of PSO and $A B C$ for abnormal brain detection [J].International Journal of Imaging Systems and Technology, 2015.25(2): p. 153-164.

[20] Zhang, Y., et al., Detection of Alzheimer's disease and mild cognitive impairment based on structural volumetric MR images using 3D-DWT and WTA-KSVM trained by PSOTVAC [J].Biomedical Signal Processing and Control, 2015.21: p. 58-73.

[21] Zhang, Y., et al., Detection of subjects and brain regions related to Alzheimer's disease using 3D MRI scans based on eigenbrain and machine learning [J].Frontiers in Computational Neuroscience, 2015.66(9): p. 1-15. 